\title{
The Pricing of Short-Lived Options \\ When Price Uncertainty \\ Is Log-Symmetric stable
}

by J. Huston McCulloch

NBER and Boston College

\author{
Boston College \\ Department of Economics \\ Working Paper \#89
}

July 1978

(Corrected November 1978)

The author is particularly grateful to John Cox for stimulating criticisms of an earlier, less rigorous, approach to this problem. This work was supported by NSF-ASRA Grant No. APR76-02511.

This paper also appears as NBER Working Paper Number 264. 
THE PRICING OF SHORT-LIVED OPTIONS WHEN PRICE

UNCERTAINTY IS LOG-SYMMETRIC STABLE

J. Huston McCulloch

\begin{abstract}
The well-known option pricing formula of Black and Scholes depends upon the assumption that price fluctuations are log-normal. However, this formula greatly underestimates the value of options with a low probability of being exercised if, as appears to be more nearly the case in most markets, price fluctuations are in fact symmetric stable or log-symmetric stable. This paper derives a general formula for the value of a put or call option in a general equilibrium, expected utility maximization context. This general formula is found to yield the Black-Scholes formula for a wide variety of underlying processes generating log-normal price uncertainty. It is then used to derive the value of a short-lived option for certain processes that generate log-symmetric stable price uncertainty. Our analysis is restricted to short-lived options for reasons of mathematical tractability. Nevertheless, the formula is useful for evaluating many types of risk.
\end{abstract}

\author{
J. Huston McCulloch \\ Department of Economics \\ Boston College \\ Chestnut Hill, MA 02167 \\ 617/969-0100, Ext. 3686
}




\section{Introduction}

The famous option pricing model of Black and Scholes (1973) relies on the strong assumption that stochastic price fluctuations are log-normal, so that in continuous time the path of the logarithm of the price is almost surely everywhere continuous. This continuity enables them to exploit an arbitrage consideration to obtain a formula for the price of a put or call option that depends only on the current price, the contractual execution price, the rate of accumulation of variance, and the riskless rate of return.

However, in many markets the observed distribution of price changes is far too fat-tailed or leptokurtic to have been drawn from a normal distribution. This led Benoit Mandelbrot (1960, 1963) to propose the Paretian stable distribution as an alternative to the conventional normal assumption. Like the normal, these distributions are bell-shaped, with an infinite negative tail. However, negative prices can be ruled out by postulating that it is the changes in the logarithm of the price, rather than the changes in the price itself, that follow a stable distribution.

Fama (1965), Roll (1970), Dusak (1973), and Cornell and Dietrich (1978) have found evidence supporting Mandelbrot's stable hypothesis in the context of corporate stock, U.S. Treasury bill, commodity futures contract, and forward foreign exchange prices, respectively. The most general stable distribution may be skewed, but most of these observers have found that the data are consistent with the simplifying assumption of symmetry or log-symmetry. Fama and Roll (1968, 1970) have developed 
simple procedures for estimating the parameters of symmetric stable distributions. DuMouche1 $(1973,1975)$ has developed more sophisticated (and expensive) maximum likelihood procedures for estimating the parameters of general stable distributions, along with previously unavailable measures of the sampling errors of these estimates. Unfortunately, the logic of the Black-Scholes model cannot be applied to stable processes because the sample paths of the latter are full of discontinuities. In fact, in any finite time interval there are almost surely an infinite number of discontinuities. Thus the crucial arbitrage consideration of the Black-Scholes argument is inapplicable. Cox and Ross (1976) are able to apply Black-Scholes logic to a certain class of jump processes to obtain an option pricing formula, but the class they consider is not very general. Merton (1976) uses the Sharpe-Lintner-Mossin capital asset pricing model to evaluate options on securities involving more general jump processes. However, the capital asset pricing model explicitly assumes that relative prices of consumption goods are constant, an assumption that is often violated when we are referring to price speculation itself. Fama (1970) demonstrates that if peoples' market portfolio behavior under certainty were unaffected by relative prices, then their behavior under uncertainty would be unaffected by the actual distribution of relative prices, and the conclusions of the capital asset pricing model would still be valid, in spite of the price uncertainty. However, this strikes the present author as an unacceptably strong assumption. We therefore do not expect the capital asset pricing model to be useful in the present context. 
If we are to evaluate options in a stable context, we must therefore start from scratch, and build up from expected utility maximization in a general equilibrium model of the economy. In Section II we build a general model of forward contract and option pricing in a simple two-good world with identical, risk-averse individuals. In Section III the endowments of the two goods are assumed to be bivariate log-normal, and the common utility function to be additively separable with constant relative rates of risk aversion. The considerable attention we pay to this case is motivated by a desire (prompted by a suggestion from John Cox) to relate the stable case to the Black-Scholes model. We find that the general option pricing formula we will apply to the stable case does indeed lead to the Black-Scholes formula in the normal case, for all values of the unobserved variables governing the price-generating process. Finally, in Section IV we apply our general option formula to the case of log-symmetric stable price changes. The formula we obtain rests on a certain specification of the unobserved variables generating the process governing price movements, and on the condition that the option have a short life (approaching zero), so our formula does not have the generality in the stable case that the BlackScholes approach has in the normal case. In the concluding Section $V$ we note that it does have a simple interpretation in terms of the actuarial value of the option, and is useful in many contexts. 
II. Forward Contract and Option Pricing in a Simple General Equilibrium Model

Consider a world in which there are two goods, $x$ and $y$, whose random endowments at some future time at which consumption is to take place we denote by $\tilde{x}$ and $\tilde{y}$. Everyone agrees that they have some joint probability density function defined on the non-negative quadrant, such as that illustrated in Figure 1. Each individual has an equal share in the output of these goods, and tries to maximize the expected value of some concave utility function $\mathrm{U}(\mathrm{x}, \mathrm{y})$, which is the same for each individual. The market-clearing (zero-trading) price of $x$ in terms of $y$ will then be

$$
\tilde{p}=\frac{U_{x}(\tilde{x}, \tilde{y})}{U_{y}(\tilde{x}, \tilde{y})} .
$$

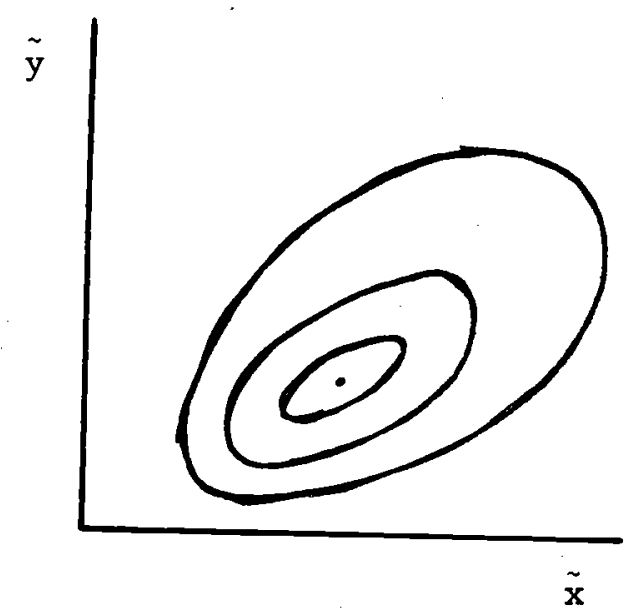

Figure 1

Price is thus a function of $\tilde{x}$ and $\tilde{y}$. Figure 2 illustrates income expansion paths along which price is constant. These lines are not necessarily straight, unless utility happens to be homothetic. However, if we plot $U_{x}$ and $\mathrm{U}_{\mathrm{y}}$ on the axes, as in Figure 3, equal price lines are straight rays from the origin, now in reverse order. If we plot this diagram logarithmically,

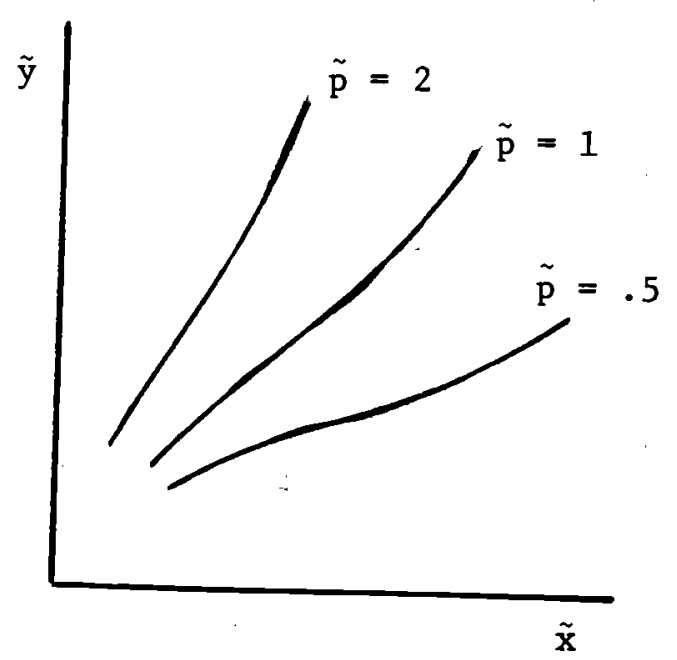

Figure 2 
the equal price lines become parallel, as in Figure 4.

Today people can enter into forward contracts to deliver one unit of $x$ at the forward price $p_{f}$. If someone were to buy $\Delta x$ units of $x$ at this forward price he would be obligated to deliver $\mathrm{p}_{\mathrm{f}} \Delta \mathrm{x}$ units of $y$. The first-order condition for utility maximization and zero-trading market clearing condition require that

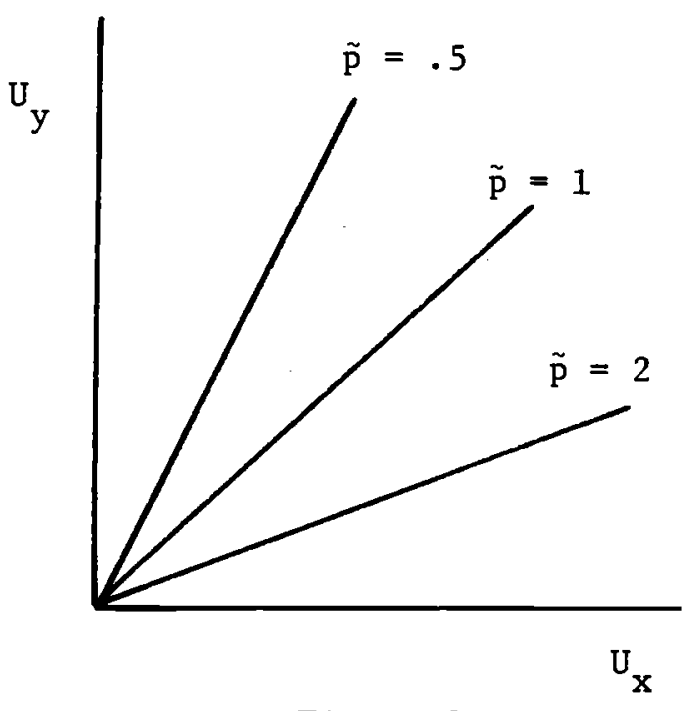

Figure 3

$$
\left.\frac{\mathrm{dEU}\left(\tilde{\mathrm{x}}+\Delta \mathrm{x}, \tilde{\mathrm{y}}-\mathrm{p}_{\mathrm{f}} \Delta \mathrm{X}\right)}{\mathrm{d} \Delta \mathrm{x}}\right|_{\Delta \mathrm{x}=0}=0,
$$

or

$$
E U_{x}(\tilde{x}, \tilde{y})=p_{f}^{E U}(\tilde{x}, \tilde{y}),
$$

so that

$$
p_{f}=\frac{E U_{x}}{E U_{y}}
$$

is the general forward contract pricing formula. Note that in general

$$
\mathrm{p}_{\mathrm{f}} \neq \mathrm{E}\left(\frac{\mathrm{U}}{\mathrm{U}}\right)
$$

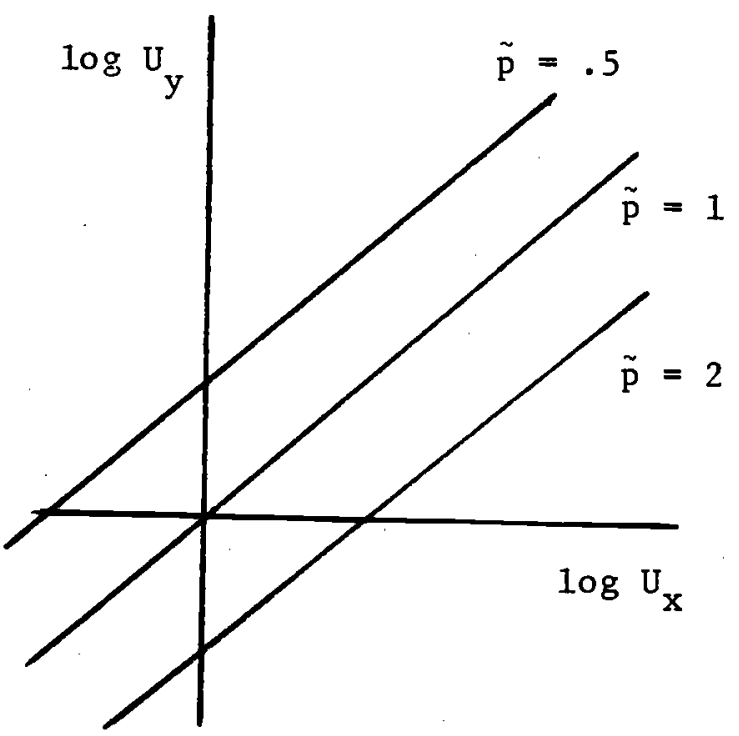

Figure 4 
There are two types of simple options, "puts" and "ca11s". A call is an option entitling one to "call forth" a certain amount of a good at a pre-arranged execution price $\mathrm{p}_{\mathrm{e}}$. It is advantageous to exercise such an option whenever $\tilde{p}>p_{e}$. One must pay $p_{c}$ for such an option. A put is an option entitling one to unload or "put off" a certain amount of a good at a pre-arranged execution price that we will again designate by $\mathrm{p}_{\mathrm{e}}$. This right will be exercised whenever $\tilde{\mathrm{p}}<\mathrm{p}_{\mathrm{e}}$ and is worth $\mathrm{p}_{\mathrm{p}} \cdot \mathrm{A}$ call on $\Delta \mathrm{x}$ units of $\mathrm{x}$ at $\mathrm{p}_{\mathrm{e}}$ is equivalent to a put on $\mathrm{p}_{\mathrm{e}} \Delta \mathrm{x}$ units of $\mathrm{y}$.

Buying a call and at the same time selling a put, both on $\Delta x$ units of $x$ at $p_{e}$ is equivalent to buying $\Delta x$ forward at price $p_{e}$. Such a forward contract would be worth $\left(p_{f}-p_{e}\right) \Delta x$, so arbitrage requires

$$
p_{f}-p_{e}=p_{c}-p_{p}
$$

If someone buys a call for $\Delta x$ units of $x$ and $\tilde{p}$ turns out to be less than $\mathrm{p}_{e}$, he will simply be out $\mathrm{p}_{c} \Delta \mathrm{x}$ units of $\mathrm{y}$ and utility will be $\mathrm{U}\left(\tilde{\mathrm{x}}, \tilde{\mathrm{y}}-\mathrm{p}_{\mathrm{c}} \Delta \mathrm{x}\right)$. If $\tilde{\mathrm{p}}>\mathrm{p}_{\mathrm{e}}$, utility will be $\mathrm{U}\left(\tilde{\mathrm{x}}+\Delta \mathrm{x}, \tilde{\mathrm{y}}-\left(\mathrm{p}_{\mathrm{c}}+\mathrm{p}_{\mathrm{e}}\right) \Delta \mathrm{x}\right)$. The first-order condition for expected utility maximization with respect to $\Delta x$, evaluated at the market-clearing condition $\Delta x=0$, now yields

$$
\begin{gathered}
\int_{\tilde{p}>p_{e}} U_{x} d P(\tilde{x}, \tilde{y})-\int_{\tilde{p}>p_{e}}\left(p_{c}+p_{e}\right) U_{y} d P(\tilde{x}, \tilde{y}) \\
-\int_{\tilde{p}<p_{e}} p_{c} U_{y} d P(\tilde{x}, \tilde{y})=0,
\end{gathered}
$$

or 


$$
P_{c}=\frac{\int_{\tilde{p}>p_{e}} U_{x} d P-p_{e} \int_{\tilde{p}>p_{e}} U_{y} d P}{E U_{y}} .
$$

Utilizing (1), we have the general formula for the price of a call option:

$$
P_{c}=\frac{P_{f}}{E U_{x}} \int_{\tilde{p}>P_{e}} U_{x} d P-\frac{p_{e}}{E U_{y}} \int_{\tilde{p}>p_{e}} U_{y} d P .
$$

Similar reasoning yields the general value of a put option:

$$
p_{p}=\frac{p_{e}}{E U_{y}} \cdot \int_{\tilde{p}<p_{e}} U_{y} d P-\frac{p_{f}}{E U_{x}} \int_{\tilde{p}<p_{e}} U_{x} d P .
$$

Equations (3) and (4) satisfy the arbitrage condition (2). 
III. The Normal Case

Now let us assume that $\tilde{x}$ and $\tilde{y}$ are bivariate log-normal such that

$$
\operatorname{Cov}(\log \tilde{x}, \log \tilde{y})=\left(\begin{array}{lr}
\sigma^{2} & \sigma \sigma \tau \\
\rho \sigma \tau & \tau^{2}
\end{array}\right) .
$$

Assume also that utility has the particular form

$$
U(\tilde{x}, \tilde{y})=\frac{1}{1-a} \tilde{x}^{1-a}+\frac{1}{1-b} \tilde{y}^{1-b}
$$

The positive constants $a$ and $b$ are the (Pratt-Arrow) constant relative rates of risk aversion for gambles involving $\mathrm{x}$ and $\mathrm{y}$ respectively. This form implies

$$
\mathrm{U}_{\mathrm{x}}=\tilde{\mathbf{x}}^{-\mathrm{a}}
$$

and

$$
\mathrm{U}_{\mathrm{y}}=\tilde{\mathrm{y}}^{-\mathrm{b}}
$$

or

$$
\tilde{\xi}=\log U_{x}=-a \log \tilde{x}
$$

and

$$
\tilde{n}=\log U_{y}=-b \log \tilde{y} .
$$

Our two assumptions together have the convenient implications that $U_{x}$ and $U_{y}$, and therefore $\tilde{p}$, are also log-normal. Define

$$
\tilde{\pi}=\log \tilde{\mathrm{p}}=\tilde{\xi}-\tilde{n}
$$

and note that 


$$
\begin{aligned}
\operatorname{Cov}(\tilde{\xi}, \tilde{n}) & =\left(\begin{array}{ll}
a^{2} \sigma^{2} & \rho a b \sigma \tau \\
\rho a b \sigma \tau & b^{2} \tau^{2}
\end{array}\right) \\
\bar{\pi} & =\bar{\xi}-\bar{n}
\end{aligned}
$$

and

$$
v^{2}=\operatorname{var}(\hat{\pi})=a^{2} \sigma^{2}-2 \rho a b \sigma \tau+b^{2} \tau^{2}
$$

Let $z$ be a log-normal variable such that $\zeta=\log z$ has mean $\bar{\zeta}$ and variance $s^{2}$. Then it is well known that

$$
\begin{aligned}
\int_{0}^{z_{0}} z \operatorname{dP}(z) & =e^{\bar{\zeta}+s^{2} / 2} N(d), \\
\int_{z_{0}}^{\infty} z \operatorname{dP}(z) & =e^{\bar{\zeta}+s^{2} / 2}(1-N(d)) \\
& =e^{\bar{\zeta}+s^{2} / 2} N(-d),
\end{aligned}
$$

and

$$
E z=\int_{0}^{\infty} z \operatorname{dP}(z)=e^{\bar{\zeta}+s^{2} / 2}
$$

where $N(d)$ is the cumulative standard normal distribution function and

$$
\begin{aligned}
d & =\frac{\log z_{0}-\bar{\zeta}-s^{2}}{s} \\
& =\frac{\log z_{0}-\log E z-s^{2} / 2}{s} .
\end{aligned}
$$

These identities have been exploited by Case M. Sprenkle (1964) and others, in the context of option pricing. 


$$
\begin{aligned}
& \text { Equations (1), (12), (13) and (16) imply } \\
& \mathrm{p}_{\mathrm{f}}=\mathrm{e}^{\bar{\pi}+\frac{1}{2}\left(\mathrm{a}^{2} \sigma^{2}-\mathrm{b}^{2} \tau^{2}\right)} \text {. }
\end{aligned}
$$

Note that $a^{2} \sigma^{2}=b^{2} \tau^{2}$ implies

$$
\log \mathrm{p}_{\mathrm{f}}=\mathrm{E} \log \tilde{\mathrm{p}}
$$

Osborne (1964) based his log-martingale model for prices on the assumption of logarithmic utility $(a=b \rightarrow 1)$. We can now see that this is unnecessarily strong. A martingale in logarithms results whenever $\mathbf{x}$ and $y$ make equal contributions to price uncertainty ( $\operatorname{var} \log U_{x}=\operatorname{var} \log U_{y}$ ), a condition which depends in part on the relative values of $a$ and $b$, but not on their absolute values. A further implication is that the levels martingale

$$
\mathrm{p}_{\mathrm{f}}=\mathrm{E} \tilde{\mathrm{p}}
$$

postulated by Samuelson and others is only a special case (arising when $\tau^{2}=0, b=0$, or $\rho=+1$, and a rather extreme special case at that. In particular, Samuelson's "theorem of the virtual certainty of (relative) ruin" (1965, p. 17) is valid only if $a^{2} \sigma^{2}>b^{2} \tau^{2}$. Note also that the Boyer inequality $(1972,1977)$,

$$
1 / E(1 / \tilde{p}) \leqq p_{f} \leqq E \tilde{p},
$$

holds whenever $\rho \leqq 0$. (with equality obtaining only if $a, b, \sigma^{2}$ or $\tau^{2}$ is zero). We could have

$$
E \tilde{p} \leqq p_{f}
$$


on $1 y$ if $\rho \gg 0$ and $a^{2} \sigma^{2} \gg b^{2} \tau^{2}$, and

$$
\mathrm{P}_{\mathrm{f}} \leqq 1 / \mathrm{E}(1 / \tilde{\mathrm{p}})
$$

only if $\rho \gg 0$ and $a^{2} \sigma^{2} \ll b^{2} \tau^{2}$.

To evaluate our call price

formula (3) we need to integrate

$U_{x} d P$ and $U_{y} d P$ over states of the world in which $\tilde{\mathrm{p}}>\mathrm{P}_{\mathrm{e}}$. These states are illustrated by the shaded area in Figure 5, where $\mathrm{p}_{\mathrm{e}}$ is arbitrarily shown as being greater than $\mathrm{p}_{f}$. This calculation is simplified by noting that $\tilde{\xi}$ and $\tilde{\pi}=\log \mathrm{p}$ are bivariate normal, with

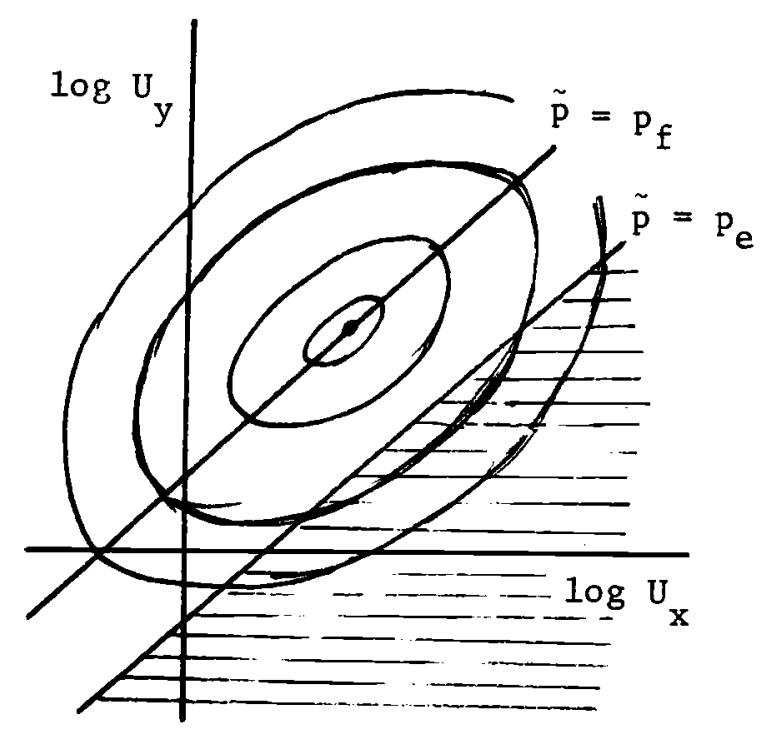

Figure 5

$$
\operatorname{Cov}(\tilde{\xi}, \tilde{\pi})=a^{2} \sigma^{2}-\rho a b \sigma \tau .
$$

For convenience, define

$$
\theta=\left(a^{2} \sigma^{2}-\rho a b \sigma \tau\right) / v^{2},
$$

so that

$$
\pi_{f}=\log P_{f}=\bar{\pi}+\left(\theta-\frac{1}{2}\right) v^{2}
$$

and

$$
\operatorname{Cov}(\tilde{\xi}, \tilde{\pi})=\theta v^{2} .
$$

Note that 
12

$$
\begin{aligned}
E(\tilde{\xi} \mid \tilde{\pi}) & =\bar{\xi}+\frac{\operatorname{cov}(\tilde{\xi}, \tilde{\pi})(\tilde{\pi}-\bar{\pi})}{\operatorname{var} \tilde{\pi}} \\
& =\bar{\xi}+\theta(\tilde{\pi}-\bar{\pi})
\end{aligned}
$$

and

$$
\begin{aligned}
\operatorname{var}(\tilde{\xi} \mid \tilde{\pi}) & =\operatorname{var} \bar{\xi}-\frac{\operatorname{cov}^{2}(\tilde{\xi}, \tilde{\pi})}{\operatorname{var} \tilde{\pi}} \\
& =a^{2} \sigma^{2}-\theta^{2} v^{2} .
\end{aligned}
$$

Equation (16) now implies that

$$
\begin{aligned}
E\left(U_{\mathbf{x}} \mid \tilde{\pi}\right) & =e^{E(\tilde{\xi} \mid \tilde{\pi})} e^{\frac{1}{2} \operatorname{var}(\tilde{\xi} \mid \tilde{\pi})} \\
& =e^{\bar{\xi}+\frac{1}{2}\left(\mathrm{a}^{2} \sigma^{2}-\theta^{2} v^{2}\right)} e^{\theta(\tilde{\pi}-\bar{\pi})}
\end{aligned}
$$

is a log-normal variable. Set $\pi_{e}=\log \mathrm{p}_{\mathrm{e}}$ and $\tilde{\zeta}=\theta(\tilde{\pi}-\bar{\pi})$. Note that $\tilde{\zeta}$ is normal with mean 0 and variance $\theta^{2} v^{2}$. We then have, with the help of (15),

$$
\begin{aligned}
\int_{\tilde{p}>P_{e}} U_{x} d P(\tilde{x}, \tilde{y}) & =\int_{\tilde{\pi}>\pi} E\left(U_{x} \mid \tilde{\pi}\right) d P(\tilde{\pi}) \\
& =\int_{\theta(\tilde{\pi}-\bar{\pi})}>\theta\left(\pi e^{-\bar{\pi})} e^{\bar{\xi}+\frac{1}{2}\left(a^{2} \sigma^{2}-\theta^{2} v^{2}\right)} e^{\theta(\tilde{\pi}-\bar{\pi})} d P(\tilde{\pi})\right. \\
& =e^{\bar{\xi}+\frac{1}{2}\left(a^{2} \sigma^{2}-\theta^{2} v^{2}\right)} \int_{\tilde{\zeta}^{\prime}>\theta(\pi} e^{-\bar{\pi})} e^{\tilde{\zeta}} d P(\tilde{\zeta}) \\
& =e^{\bar{\xi}+\frac{1}{2}\left(a^{2} \sigma^{2}-\theta^{2} v^{2}\right)} e^{\theta^{2} v^{2} / 2} N\left(-d_{1}^{*}\right)
\end{aligned}
$$




$$
\begin{aligned}
& =e^{\bar{\mu}+\frac{1}{2} a^{2} \sigma^{2}} N\left(-d_{1}^{*}\right) \\
& =E U_{x} N\left(d_{1}\right)
\end{aligned}
$$

where

$$
\begin{aligned}
d_{1}^{*} & =\frac{\theta\left(\pi e^{-\bar{\pi})}-\theta^{2} v^{2}\right.}{\theta v} \\
& =\frac{\pi_{e}-\bar{\pi}-\theta v^{2}}{v} \\
& =\frac{\pi_{e}-\pi_{f}-\frac{1}{2} v^{2}}{v},
\end{aligned}
$$

so that

$$
d_{1}=-d_{1}^{*}=\frac{\log \left(p_{f} / p_{e}\right)+\frac{1}{2} v^{2}}{v} .
$$

By similar reasoning,

$$
\int_{\tilde{p}>P_{e}} U_{y} d P(\tilde{x}, \tilde{y})=E U_{y} N\left(d_{2}\right),
$$

where

$$
d_{2}=\frac{\log \left(p_{f} / p_{e}\right)-\frac{1}{2} v^{2}}{v} .
$$

Combining these results with (3) yields

$$
p_{c}=p_{f} N\left(d_{1}\right)-p_{e} N\left(d_{2}\right)
$$

Similar reasoning (or the arbitrage condition (2)) yields

$$
p_{p}=p_{e} N\left(-d_{2}\right)-p_{f} N\left(-d_{1}\right)
$$


Equations (31) and (32) are essentially the Black-Scholes option pricing formulas. The full Black-Scholes formulas emerge if we set $v^{2}=v_{0}^{2} t$ and discount $p_{c}, p_{p}$, and $p_{f}$ to the present at the riskless own interest rate on $\mathrm{y}$-denominated loans. (The current price of a costlessly stored asset that provides no current services is its discounted forward price. Furthermore, we developed (3) and (4) as if payment for the option were to be made on the execution date, rather than the present date as is conventional.) Note that these formulas only involve $P_{f}, P_{e}$, and $v^{2}$, the variance of $\log \tilde{p}$. They do not depend on $a, b, \sigma^{2}, \tau^{2}$, or $\rho$, which is convenient, since these variables cannot be inferred from price behavior alone. The summary variable $\theta$ could be inferred from the relation between $P_{f}$ and $E \log \tilde{p}$, but even it drops out.

The model from which we have derived (31) and (32) is perhaps not the most general that produces log-normal price behavior with two consumption goods. The assumption of a constant relative rate of risk aversion made the 1 log-normality of $\tilde{x}$ and $\tilde{y}$ carry over to $U_{x}$ and $U_{y}$, but may not be necessary. Certain forms of interaction between $U_{x}$ and $U_{y}$ merely alter the (immaterial) correlation coefficient between $\tilde{\xi}$ and $\tilde{\eta}$ and therefore do not disturb our formulas. But other, more complicated, interactions may still produce log-normal prices, yet require new reasoning. Although our model is not completely general in the lognormal case, it does cover enough different variations on the underlying process that we believe it is at least approximately valid in all cases leading to log-normal prices of consumption goods.

Two important markets do not fit our model precisely. First is the foreign exchange market, where it is more realistic to postulate 
one aggregate consumption good and random (log-norma1) price levels in both countries, with the exchange rate determined by purchasing power parity. This is the model of Boyer (1972, 1977). Second is a "stock" market, in which the price in question is the price of shares in the total output of a single consumption good. In spite of the dissimilarity between these markets and the one we have modelled, we would not be surprised to find that (31) and (32) remain valid.

An equation like (31) was first obtained by Sprenkle (1964, p. 434) as the actuarial value of an option, when the present price equals the expected future price. He quickly dismissed the (valid) simple form as allowing no compensation for risk aversion, and insisted that it should instead be adjusted by an arbitrary risk factor. The actuarial formula turns out to have been valid after all. When $b=0$ or $\tau^{2}=0$, actuarial calculations are valid since $U_{y}$ is then constant. The formula remains valid when $b$ and $\tau^{2}$ are not zero, since their values are immaterial. Apparently the $p_{f}$ that appear in the formulas already contains all the adjustment for risk aversion we need to make.

The novelty of the Black-Scholes model is not, therefore, so much in the formula itself, but rather in the claim that the naive actuarial Sprenkle formula is actually universally valid, at least for log-normal processes. While we concur with its validity, it is not clear to the present author why their reasoning should establish that validity. Their argument rests on the assertion that a portfolio whose price cannot fluctuate is riskless. Yet it is clear from our model that utility can fluctuate while price remains constant, as $\tilde{x}$ and $\tilde{y}$ move along one of the income-expansion paths of Figure 2. Furthermore, price can 
fluctuate while utility remains constant, as $\tilde{x}$ and $\tilde{y}$ move along an indifference curve. It may be that the validity of the Black-Scholes formula, like that of the (essentially identical) actuarial Sprenkle formula, derives from the case $U_{y}=$ constant, in which event their reasoning is definitely valid.

IV. The Log-Symmetric Stable Case

We have seen that the Sprenkle/Black-Scholes formula apparently covers the field when price variation is log-normal. However, it will generally underestimate the value of an option with low probability of exercise when (as appears to be more nearly the case empirically) price variation is log-symmetric stable. In this case we must resort to the basic option pricing formulas (3) and (4). Our somewhat lengthy discussion of the log-normal case was necessary in order to establish the consistency of these formulas with the pre-eminent Black-Scholes formula.

If $\tilde{\mathbf{x}}$ and $\tilde{y}$ are log-stable and utility exhibits constant relative rates of risk aversion as in (5), price will also be log-stable. There is a covariability concept for stable distributions, analogous to covariance when variance is finite, but since our $\rho$ did not affect the normal case, we will assume that $\log \tilde{\mathrm{x}}$ and $\log \tilde{\mathrm{y}}$ are independent stable variables.

If $\log \tilde{\mathbf{x}}$ and $\log \tilde{y}$ were themselves symmetric stable variables, and the relative rates of risk aversion were strictly constant as in (6) 
and (7), the denominators and at least two of the numerators in the right hand sides of (3) and (4) would be infinite, so that evaluating them would involve problemmatic ratios of infinities. We could easily eliminate this problem by postulating any bounded utility function à 1a Karl Menger, but then the stability of $\log \tilde{\mathbf{x}}$ and $\log \tilde{y}$ would no longer carry over to $\log U_{x}$ and $\log U_{y}$, so that $\log \tilde{p}$ might not be precisely stable. More radically, we could challenge the whole rationale for expected utility maximization (e.g. Meginniss, 1977).

What we will do instead is make the special assumption that $\log \tilde{\mathrm{x}}$ and $\log \tilde{\mathrm{y}}$ are not symmetric stable (skewness parameter $\beta$ equal to 0 ), but rather are maximally positively skewed $(\beta=+1)$. In an early article Mandelbrot (1960) actually proposed these maximally skewed stable variables, rather than the symmetric case, as the premier model for economic variables. Equations (8) and (9) then imply that $\tilde{\xi}$ and $\tilde{n}$ are maximally negatively skewed $(\beta=-1)$. When $\beta=-1$, the upper tail of a stable distribution completely loses its Paretian component. The upper tail is still infinite, but it falls off even more rapidly than the upper tail of a Gaussian distribution, so that $\mathrm{EU}_{\mathrm{x}}$ and $\mathrm{EU}_{\mathrm{y}}$ become finite. If $\tilde{\xi}$ and $\tilde{\eta}$ are skewed, then $\tilde{\pi}$, in accordance with (12), will be somewhat less skewed. It can be exactly symmetric, but only if the variability of $\tilde{\xi}$ and $\tilde{n}$ are equal. This is analogous to the case $a \sigma^{2}={ }^{k} \tau^{2}$ in the normal case. As in the normal case, we will then have the "log-metric" forward price of equation (19). Note once again that this Osbornian log-random walk does not require logarithmic utility, but only that the contributions to price variability from $U_{x}$ and $U_{y}$ be equal. We will assume this is approximately true, in order to generate 
the observed approximate symmetry of $\tilde{\pi}$, given our assumption that $\tilde{\xi}$ and $\tilde{n}$ are maximally skewed.

When variability is finite, the stable distribution is defined in terms of the inverse Fourier transform of its characteristic function, which is unenlightening to most non-mathematicians, including the present author. However, in continuous time, when the variability over one instant goes to zero, it becomes more tractable. Let $c_{0}$ be the "standard scale" that accumulates in 1.0 time unit in a continuous time process with independent symmetric stable increments, so that $c=c_{0} t^{1 / \alpha}$ is the scale that accumulates in $t$ time units. Such a process consists of a linear drift plus the sum of a number of discontinuities. The probability that a discontinuity greater than $x_{0}>0$ (or less than $-x_{0}$ ) in size will not occur in $t$ time units is $e^{-\lambda t}$, where the average rate of occurrence $\lambda$ is given by the formula

$$
\lambda=\frac{\mathrm{k}_{\alpha}}{2}\left(\frac{{ }^{c_{0}}}{\mathrm{x}_{0}}\right)^{\alpha} .
$$

The constant $\mathrm{k}_{\alpha}$ is defined by

$$
\mathrm{k}_{\alpha}=\frac{2}{\pi} \Gamma(\alpha) \sin \left(\frac{\pi \alpha}{2}\right),
$$

and is tabulated in McCulloch (1978b, ms. p. 19), where these formulas are derived. When a discontinuity greater than $x_{0}$ (less than $-x_{0}$ ) does occur, the probability that it is less than $\mathbf{x}$ (greater than $-\mathbf{x}$ ) is governed by the simple Pareto distribution (whence the "Paretian" character of the stable distributions): 


$$
\mathrm{F}_{\alpha}\left(\mathrm{x} ; \mathrm{x}_{0}\right)= \begin{cases}0 & x<\mathrm{x}_{0} \\ 1-\left(\frac{\mathrm{x}_{0}}{\mathrm{x}}\right)^{\alpha} & \mathrm{x} \geqq \mathrm{x}_{0}\end{cases}
$$

This has density

$$
f_{\alpha}\left(x ; x_{0}\right)= \begin{cases}0 & x<x_{0} \\ \alpha x_{0}^{\alpha} x^{-\alpha-1} & x \geq 0\end{cases}
$$

Consider now a continuously renewable option, which has life $d t \rightarrow 0$ (so that $p_{f}$ becomes the current spot price $p_{s}$ ) and in which the ratio $\mathrm{r}=\mathrm{p}_{\mathrm{e}} / \mathrm{p}_{\mathrm{s}}$ is held constant. Such a call option with $\mathrm{r}<1$ would have infinite value per unit time since it could be executed each instant for a profit of roughly $\mathrm{p}_{s}-\mathrm{P}_{\mathrm{e}}$ every time. Similarly, a put option with $r>1$ would have infinite value per unit time. We therefore assume $r>1$ for call options, and $r<1$ for put options.

Let us take the particular case of a continuously renewable put option with life $\mathrm{dt}$, and with $\mathrm{r}<1$. The price $\tilde{\mathrm{p}}$ may fluctuate, but $\mathrm{P}_{\mathrm{e}}$ is immediately adjusted to return $r$ to its initial value. The only way it can become desirable to exercise such an option is for $\tilde{p}$ to fall below $\mathrm{P}_{\mathrm{e}}$ through a (negative) discontinuity in $\pi$ smaller in algebraic value than $\log \mathrm{r}$. Letting $\mathrm{c}_{0}$ be the standard scale of $\tilde{\pi}$ that accumulates in 1.0 year, the rate of occurrence of such discontinuities is

$$
\lambda=\frac{k_{\alpha}}{2}\left(\frac{c_{0}}{-\log r}\right)^{\alpha}
$$

In order to evaluate (4), we must investigate the origins of the discontinuities. Since a maximally skewed stable variable has no Paretian component in its thin tail, a continuous time process with 
independent maximally skewed stable increments only has discontinuities in the direction of the fat Paretian tail, with drift in the opposite direction to balance the discontinuities. Such processes are illustrated by Cross (c 1975). Since $\log \mathrm{U}_{\mathrm{x}}$ and $\log \mathrm{U}_{\mathrm{y}}$ are each maximally negatively skewed, positive jumps in $\mathrm{P}$ must be coming from $\mathrm{U}_{\mathrm{y}}$, and negative jumps from $U_{x} \cdot$ Both types are occurring with frequency $\lambda$ as in (36), but only the negative ones ( $f r o m U_{x}$ ) can cause $\tilde{p}<P_{e}$ with a continuously renewable put. We therefore have

$$
\begin{aligned}
\int_{\tilde{\mathrm{P}}<\mathrm{p}_{e}} U_{y} d P(\tilde{x}, \tilde{y}) & =E\left(U_{y} / \tilde{\mathrm{p}}<\mathrm{P}_{e}\right) \cdot \lambda d t \\
& =E U_{y} \cdot \lambda d t
\end{aligned}
$$

and, setting $\tilde{\zeta}=\bar{\xi}-\tilde{\xi}$,

$$
\begin{aligned}
& \int_{\tilde{\mathrm{p}}<\mathrm{p}_{e}} U_{\mathrm{x}} \mathrm{dP}(\tilde{\mathrm{x}}, \tilde{\mathrm{y}})=E\left(U_{x} / \tilde{\mathrm{p}}<\mathrm{p}_{\mathrm{e}}\right) \cdot \lambda \mathrm{dt} \\
& =\int_{-\infty}^{\bar{\xi}+\log r} e^{\tilde{\xi}} f_{\alpha}(\bar{\xi}-\tilde{\xi} ;-\log r) d \tilde{\xi} \cdot \lambda d t \\
& =e^{\bar{\xi}} \int_{-\log r}^{\infty} e^{-\tilde{\zeta}} f_{\alpha}(\tilde{\zeta} ;-\log r) d \tilde{\zeta} \cdot \lambda d t .
\end{aligned}
$$

As in the log-normal case, we have

$$
E U_{x}=e^{\bar{\xi}}(1+o(d t))
$$

where $o(d t)$ indicates an unspecified function such that $\lim _{d t \rightarrow 0} \frac{o(d t)}{d t}$ is bounded. 
Putting together (4), (37), (38) and (39), we obtain

$$
P_{p}=P_{e} \lambda d t-\frac{p_{s}}{1+o(d t)} \int_{-\log r}^{\infty} e^{-\tilde{\zeta}} f_{\alpha}(\tilde{\zeta} ;-\log r) d \tilde{\zeta} \cdot \lambda d t
$$

The term in o(dt) leads only to terms in o(dt $\left.{ }^{2}\right)$, so we may eliminate it. We then have the following expression for the value per unit time of a short-lived put, as a fraction of the current price of the good or asset in question:

$$
\frac{1}{d t} \frac{p_{p}}{p_{s}}=\lambda H(r, \alpha)
$$

where

$$
H(r, \alpha)=r-\int_{-\log r}^{\infty} e^{-\tilde{\zeta}} f_{\alpha}(\tilde{\zeta} ;-\log r) d \tilde{\zeta}
$$

and

$$
r=\frac{p_{e}}{p_{s}} \cdot \underline{1 l}
$$

The function $H(r, \alpha)$ depends on the integral in (42), which is the expected reciprocal of a log-Pareto variable. This integral must be evaluated numerically, which we have done with the computational assistance of Tom Mroz. Selected values of this function are indicated in Table 1. They should be accurate to the precision shown.

1/ In an earlier paper (1978a) the present author used a heuristic argument to evaluate a short-lived put as $\lambda \log (1 / r) /(\alpha-1)$, which, as can be shown, turns out to have the same behavior as (41) in the limit $r \rightarrow 1$. However, for $r<1$, the present formula gives a somewhat lower value. 


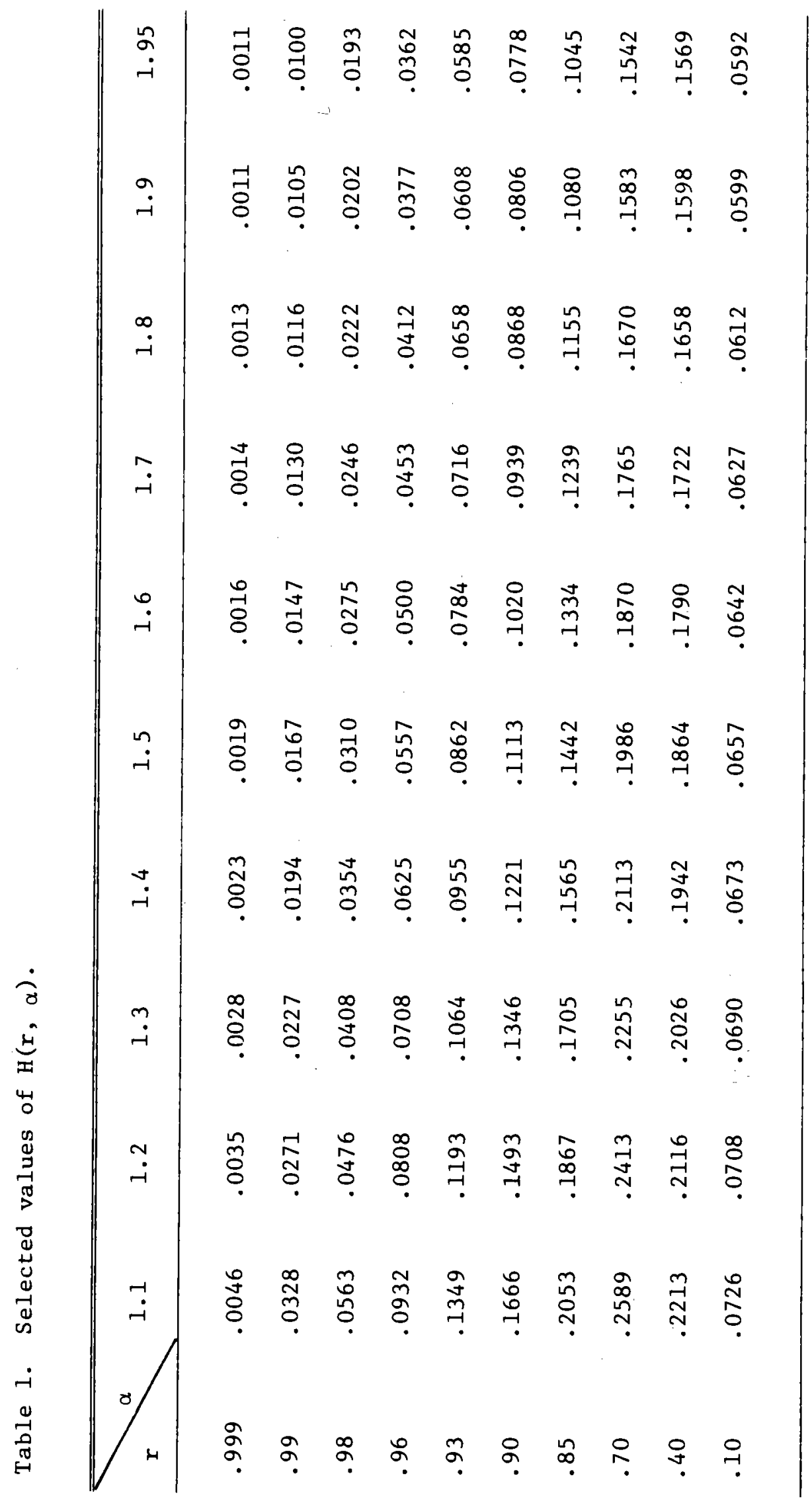


By analogous reasoning using (3), or by interchanging the roles of $\mathrm{x}$ and $\mathrm{y}$ and observing that a put on $\mathrm{y}$ is the same contract as a call on $x$, we have the following formula for the value per unit time of a continuously renewed call option:

$$
\frac{1}{d t} \frac{P_{c}}{P_{s}}=\lambda H(r, \alpha),
$$

where now

$$
r=\frac{\mathrm{P}_{\mathrm{s}}}{\mathrm{P}_{\mathrm{e}}} \text {. }
$$

\section{Conclusion}

Although our put pricing formula has a somewhat roundabout derivation, its interpretation is quite simple: It is simply the actuarial value of the option, given the good's forward price $p_{f}$ (which has converged on $\mathrm{p}_{\mathrm{s}}$ ). In this respect it resembles the Sprenkle/BlackScholes model. Note, however, that while the latter can be interpreted as fully actuarial, with $\mathrm{p}_{\mathrm{f}}=\mathrm{E} \tilde{\mathrm{p}}$, in our formula we have $\mathrm{p}_{\mathrm{f}}=\mathrm{e}^{\mathrm{E} \log \tilde{\mathrm{P}}}<\mathrm{E} \tilde{\mathrm{p}}=\infty$.

In a stable world, the actuarial value of a call, even a shortlived one, is infinite. However, this is irrelevant, since in the case of a short-lived call, the appropriate valuation formula is actuarial in reciprocal prices (the price of $y$ in terms of $x$ ). (The Sprenkle/BlackScholes formula has the remarkable property that it is simultaneously fully actuarial in prices and reciprocal prices.) 
The assumption of a short option life undoubtedly restricts the precision of our formula for highly speculative options with a moderate to high probability of execution. We see its chief application as the evaluation of default risk on the part of relatively safe firms with limited liability, such as banks, commercial corporations, and imperfectly covered commodity and stock brokerage firms, and of non-performance risk on speculative options. Perhaps others can extend our results to more risky options, and to more general underlying processes. 


\section{BIBLIOGRAPHY}

Black, F. and M. Scholes, "The Pricing of Options and Corporate Liabilities," J. Politica1 Economy 83 (1973), 637-659.

Boyer, R. S., "The Interest Rate Parity Theorem Again: A Resolution of the Siegel Paradox," mimeo, Univ. of Western Ontario, Sept. 1972.

, "The Relation between the Forward Exchange Rate and the Expected Future Spot Rate," Intermountain Economic Review 8 (Spring 1977), 14-21.

Cootner, P. H. (ed.), The Random Character of Stock Market Prices (Cambridge: M.I.T. Press, 1964).

Corne11, W. B. and J. K. Dietrich, "The Efficiency of the Market for Foreign Exchange under Floating Exchange Rates," Review of Economics and Statistics 60 (Feb. 1978), 111-120.

Cox, J. C. and S. A. Ross, "The Valuation of Options for Alternative Stochastic Processes," J. Financial Economics 3 (1976), 145-166.

Cross, M. J., "A Catalogue of Finite Mean Stable Random Walks," mimeo, Bell Laboratories, Murray Hill, N.J., c. 1975.

DuMouche1, W. H., "On the Asymptotic Normality of the Maximum-Likelihood Estimate when Sampling from a Stable Distribution," Annals of Statistics 1 (1973), 948-957.

, "Stable Distributions in Statistical Inference: 2. Information from Stably Distributed Samples," J. American Statistical Assn. 70 (June 1975), 386-393.

Dusak, K., "Futures Trading and Investor Returns: An Investigation of Commodity Market Risk Premiums," J. Political Economy 81 (Nov./Dec. 1973), 1387-1406.

Fama, E. F., "The Behavior of Stock Market Prices," J. Business 38 (Jan. 1965), 34-105.

, "Multiperiod Consumption-Investment Decisions," American Economic Review 60 (March 1970), 163-174.

and Roll, R., "Some properties of Symmetric Stable Distributions," J. American Statistical Assn. 63 (Sept. 1968), 817-836.

and , "Parameter Estimates for Symetric Stable Distributions," J. American Statistical Assn. 66 (June 1971), 331-338. 
Mandelbrot, B., "The Pareto-Lévy Law and the Distribution of Income," International Economic Review 1 (May 1960), 79-106.

, "The Variation of Certain Speculative Prices," J. Business 36 (Oct. 1963), 394-419. (Reprinted in Cootner (ed.), 1964, 307-332.)

McCulloch, J. H., "Interest Rate Risk and Capital Adequacy for Traditional Banks and Financial Intermediaries," NBER Working Paper 237, Jan. 1978 (1978a).

, "Continuous Time Processes with Stable Increments," J. Business 51 (Oct. 1978), forthcoming.

Meginniss, J. R., "Alternatives to the Expected Utility Rule," unpublished Ph.D. dissertation, Univ. of Chicago Graduate School of Business, 1977.

Merton, R. C., "Option Pricing when Underlying Stock Returns are Discontinuous," J. Financial Economics (1976), 125-144.

Osborne, M. F. M., "Brownian Motion in the Stock Market," in Cootner (ed.) (1964), 100-128.

Ro11, R., The Behavior of Interest Rates (New York: Basic Books, 1970).

Samuelson, P. A., "Rational Theory of Warrant Pricing," Industrial Management Review 6 (1965), 13-31.

Sprenkle, C. M., "Warrant Prices as Indicators of Expectations and Preferences," in Cootner (ed.) (1964), 412-474. 